\title{
Joyce centrífugo o la suciedad por amor al arte
}

\author{
Centrifugal Joyce, or Art for Dirt's Sake
}

JAMES RAMEY

UNIVERSIDAD AUTÓNOMA METROPOLITANa, CUajimalPa | México

Contacto: jamestramey@yahoo.com

\begin{abstract}
Resumen
En respuesta a la crítica realizada por Caren Kaplan al exilio modernista, este artículo reutiliza la teoría bajtiniana como un instrumento para comprender el cruce de fronteras y la ruptura de barreras joyceanas como formas de centrifugalidad. El trabajo también cuestiona si un novelista al que Bajtín ignoró podría haber producido la máxima expresión de los propios ideales de representación novelística de su obra: dialógico, carnavalesco, multicronotópico, corpocéntrico y lingo-culturalmente interestratificado. Para avanzar en este argumento, el principal concepto bajtiniano de heteroglosia se expande más allá de su ámbito original, intranovelista, para describir tanto las energías como las tensas complejidades del exilio como motor del arte modernista. Una propuesta de eje teórico que vincula el exilio, la heterogglosia y el formalismo ruso conduce a una discusión sobre la obscenidad como un objeto centrífugo y estético para Joyce: la suciedad por amor al arte. Y esto a su vez conduce a un análisis de los distintos niveles de representación interestratificadas en Ulysses como una forma de heteroglosia cuyo poder depende tanto del elemento exílico como del elemento afectivo. Finalmente, este artículo demuestra que Ulysses, como un alejamiento radical del lenguaje estándar y centrípeto de sus primeras novelas, representa la quintaesencia de la heteroglosia novelística, no sólo en su estilo sino también en términos espaciales: los de las fuerzas centrífugas y mutantes representadas por sus viajes por el mundo y la imagen exílica de su creador.
\end{abstract}

Palabras clave:James Joyce, Mijaíl Bajtín, exilio, heteroglosia, teoría cultural, formalismo ruso

\begin{abstract}
In response to Caren Kaplan's critique of modernist exile, this article repurposes Bakhtinian theory as an instrument for understanding Joycean border-crossing and barrier-breaking as forms of centrifugality. It also asks whether a novelist that Bakhtin ignored might turn out to have produced the maximum expression of Bakhtin's own ideals of novelistic representation: dialogical, carnivalesque, multi-chronotopic, body-centric, and lingo-culturally interstratified. To advance this argument, the major Bakhtinian concept of heteroglossia is expanded beyond its original, intranovelistic purview to describe both the energies and the fraught complexities of exile as a motor of modernist art. A proposal for a theoretical axis linking exile, heteroglossia, and Russian Formalism leads to a discussion of obscenity as both a centrifugal and an aesthetic object for Joyce: dirt for art's sake. And this in turn leads to an analysis of interstratified layers of representation in Ulysses as a form of heteroglossia that depends for its power on both the exilic and the affective. Ultimately, this article demonstrates that Ulysses, as a radical departure from the standard, centripetal language of earlier novels, represents the quintessence of novelistic heteroglossia, not only in its stylistics but also in spatial terms: those of the mutative, centrifugal forces represented by its global travels and the exilic image of its creator.
\end{abstract}

Keywords: James Joyce, Mikhail Bakhtin, exile, heteroglossia, cultural theory, Russian Formalism

${ }^{1}$ Este artículo fue traducido del inglés por Alfredo Peñuelas Rivas, en colaboración con el autor. No se ha publicado anteriormente. 
L

a formulación de Caren Kaplan sobre las economías entre la pérdida melancólica y la ganancia estética, en su renombrado Question of Travel: Postmodern Discourses of Displacement (1996), ve con "lágrimas de cocodrilo", por decirlo de alguna manera, en cada autoformulación modernista del exilio, una invención que necesita ser vista como la característica artificial que es. Sin duda, su libro explora casos significativos en los que el exilio modernista concuerda con su crítica. Sin embargo, habría que preguntarse si hay algo malo o incluso sorprendente en que un artista emplee dicho artificio para llamar la atención sobre su trabajo o su imagen. La "imagen" del artista es tanto un producto artístico como el texto que le asociamos, y puede ser interpretada e interrogada precisamente como un texto. Un ejemplo de ello sería el caso de James Joyce, un autor que Kaplan menciona sólo de pasada. En estas páginas ofrezco un contraargumento a lo anterior basado en una lectura bajtiniana de Joyce como figura exílica y de Ulysses (1990) como texto exílico. Aunque existe una tradición rica y continua de lecturas bajtinianas sobre el trabajo de Joyce, ${ }^{2}$ el enfoque que adopto aquí es ciertamente poco ortodoxo y busca replantear la teoría bajtiniana como un instrumento para comprender el cruce de fronteras y la ruptura de barreras joyceanas como formas de centrifugalidad.

En lo que respecta a los sistemas sociolingüísticos artísticos, Ulysses es un texto cuyos múltiples estratos de lenguajes heteroglóticos y viajes caleidoscópicos cronotópicos han demostrado hasta ahora ser inagotables para el análisis bajtiniano. Sin embargo, el propio Mijaíl Bajtín despreciaba notoriamente la literatura del siglo XX, especialmente las novelas occidentales modernistas. Él y otros miembros de su círculo conocían a Joyce, pero el propio Bajtín sólo lo menciona una vez, en su libro sobre Dostoievsky de 1929, cuando hace una cita aprobatoria del ataque de otro erudito soviético al "psicologismo decadente degenerado" de Joyce (y Marcel Proust) (Bajtín, 1984: 37). ${ }^{3}$ Debido a las aparentes afinidades de Joyce con el trabajo de Bajtín, especialistas como Katerina Clark y Michael Holquist (1984), además de

\footnotetext{
${ }^{2}$ Los ejemplos recientes incluyen a Azaredo (2020), Balkaya (2017), George (2017), Kim (2017), y Tekdemir (2017).

${ }^{3}$ Por cuestiones de sintaxis, las citas que originalmente estaban en inglés se presentan traducidas.
} 
R. B. Kershner (1989), han lamentado la laguna joyceana en el vasto conocimiento de sus novelas por el teórico ruso. Aunque es probable que Bajtín no haya podido publicar sobre Joyce de manera positiva debido a la política soviética, es también posible que en 1929 sintiera que el trabajo de Joyce representaba una decadencia psicológica y social después del apogeo del realismo en Dostoievski. Sin embargo, si esto último es cierto, muchos observadores estarían de acuerdo en que Bajtín, paradójicamente, habría pasado por alto las virtudes claramente bajtinianas de Joyce. ${ }^{4}$ En Ulysses particularmente, el carnaval de cronotopos en relación dialógica entre sí, su coro populoso y polifónico de voces diversas, su celebración rabelesiana del cuerpo y su asombrosa multitud de niveles de discurso interestratificados son únicos en la historia de la novela. De hecho, para muchos bajtinianos, Ulysses es el ejemplo insuperable de heteroglosia novelística. Como dice M. Teresa Caneda Cabrera (1996), el "principio heteroglósico, que explica los vínculos e interrelaciones distintivos entre enunciados y palabras, es precisamente el rasgo fundamental de la estilística de [...] Ulysses" (33-34). En este artículo, sugeriré que Ulysses, como una desviación radical del lenguaje centrípeto y estándar de las novelas anteriores, representa la quintaesencia de la heteroglosia novelística, no sólo en su estilística sino también en términos espaciales: los de las fuerzas centrífugas mutantes representadas por sus viajes por el mundo y la imagen exílica de su creador.

${ }^{4}$ Estoy muy agradecido con uno de los o las árbitras anónimas, quien informa que la evidencia publicada en ruso sugiere que Bajtín, en la víspera de su jubilación en 1960, discutió el trabajo de Joyce en términos de admiración; el o la árbitra nos traduce del ruso el siguiente comentario de las Obras en 7 tomos: "Bajtín habló en extenso de Joyce en el curso universitario de la universidad de Saransk en 1958-59 [lo cual era totalmente inusitado en su contexto], dándole un lugar muy importante [la noticia se reconstruye a partir de los apuntes de una estudiante]" (Bajtín, 1996-2012: t. 3, 812). Entonces, aunque Bajtín nunca publicó tales opiniones sobre Joyce, evidentemente por motivos de seguridad, y no queda claro si conocía su obra en 1929 cuando publicó la referencia negativa arriba mencionada, lo habría conocido para finales de la década de 1950, y en ese entonces lo tuvo en alta estima. Este grato descubrimiento hace que la propuesta teórica central de este ensayo sea todavía más pertinente. 


\section{Economías del exilio modernista}

$\mathrm{Al}$ abordar la centrifugalidad modernista con un grado mayor de escepticismo que el de los estudios anteriores, el primer capítulo de Cuestión de viaje de Kaplan (1996) apunta a las deficiencias del "giro espacial" en los estudios literarios y sostiene que este giro se desarrolló conjuntamente con el estudio (y práctica) de los “modernismos euroamericanos". Ella escribe:

Euro-American modernisms celebrate singularity, solitude, estrangement, alienation, and aestheticized excisions of location in favor of locale-that is, the "artist in exile" is never "at home," always existentially alone, and shocked by the strain of displacement into significant experimentations and insights. [...] That is, the Euro-American middle-class expatriates adopted the attributes of exile as an ideology of artistic production. (Kaplan, 1996: 28)

Kaplan (1996) critica esta ideología del exilio argumentando que "las formaciones modernistas euroamericanas del exilio fomentan una cultura de melancolía nostálgica" (34) y examina la manera en que el "aumento estético" (36) a cambio de la melancolía y la "tensión del desplazamiento" (28) ha dado forma a un tropo literario-crítico del exilio modernista. También estudia este tropo en trabajos académicos sobre el modernismo de Malcolm Bradbury, Raymond Williams, Harry Levin, Malcolm Cowley y Paul Fussell, entre otros. Kaplan (1996) identifica una veta masculinista en esta tradición y, basándose en Benedict Anderson, argumenta de manera persuasiva que la "comunidad imaginada" (29) de los modernistas, lo que Bradbury llamó la "ciudad distante ideal" (29) de los modernistas, que es "el lugar mismo de la creación del arte" (29) fue producida tanto por mujeres y por artistas en los márgenes del modernismo como por las "comunidades focales” (29) metropolitanas dominadas por hombres que Bradbury exalta. Kaplan (1996) está particularmente preocupada por la falta de perspectiva histórica en los paradigmas que estos críticos establecen para el exilio modernista: "en resumen, el exilio está completamente deshistorizado" (40). Citando Imperial Eyes de Mary Louise Pratt 
(1992), deconstruye la oposición del exilio modernista y lo que llama "turismo posmoderno" (Kaplan, 1996: 22) al vincular ambos a una genealogía del colonialismo y la conquista euroimperial.

Sin embargo, vale la pena señalar que la teoría de Kaplan también hace eco de la discusión de Bajtín sobre las fuerzas centrípetas y centrífugas del lenguaje; Kaplan (1996) escribe, por ejemplo:

Part of the configuration of contradiction and ambivalence that marks these theories of modernity includes tensions between progress and tradition. That is, modernisms promote both innovation and convention, change and stability. In modernity, cultural change is a given. Social theorists such as Marshall Berman have argued that moderns oscillate between a wholehearted endorsement of change and a deep desire for stability. If, as Berman argues, people struggle to "get a grip on the modern world and make themselves a home in it," then they also struggle to disengage or displace themselves in very deliberate and particular ways. (35)

La idea de una oscilación "entre un respaldo incondicional al cambio y un profundo deseo de estabilidad" va muy de acuerdo con la idea bajtiniana de una tensión dinámica entre monoglosia y heteroglosia, entre géneros unitarios del discurso y lenguajes de subversión. En su ensayo “Discurso y la novela”, Bajtín (1989) lo expresa de esta manera:

las fuerzas centrípetas de la vida del lenguaje, encarnadas en el "lenguaje único", actúan dentro de [una heteroglosia] efectiva. La lengua, en cada uno de los momentos de su proceso de formación, no sólo se estratifica en dialectos lingüísticos en el sentido exacto de la palabra (según los rasgos de la lingüística formal, especialmente los fonéticos), sino también -y ello es esencial- en lenguajes ideológico-sociales; de grupos sociales, "profesionales", "de género", lenguajes de las generaciones, etc. Desde este punto de vista, el mismo lenguaje literario sólo es uno más entre los lenguajes [de 
la heteroglosia], que, a su vez, está estratificado en lenguajes (de géneros, de corrientes, etc.). Esa estratificación efectiva y [la heteroglosia], no sólo constituyen la estática de la vida lingüística, sino también su dinámica: la estratificación y [la heteroglosia] se amplifican y se profundizan durante todo el tiempo en que está viva y evoluciona la lengua; junto a las fuerzas centrípetas actúan constantemente las fuerzas centrífugas de la lengua, a la vez que la centralización ideológico-verbal; con la unificación se desarrolla ininterumpidamente el proceso de descentralización y separación. (89-90)5

La tensión que describe Bajtín entre las fuerzas centrípetas, monológicas y unitarias del lenguaje (asociadas con elementos socioculturales hegemónicos) y las fuerzas centrífugas, heteroglotas y estratificadoras del lenguaje (asociadas con dialectos de grupos pequeños y jerga juvenil) forma un claro paralelo a las "tensiones entre progreso y tradición" (Kaplan, 1996: 35), a la promoción "tanto [de] la innovación como la convención, el cambio y la estabilidad" (Kaplan, 1996: 35), que Kaplan (1996) atribuye a los "modernismos" (35). En términos bajtinianos, la crítica de Kaplan al exilio modernista puede entenderse, de manera sorprendente, como una sospecha de heteroglosia.

Además, parece dudoso que Bajtín afirmara como Kaplan que "en la modernidad, el cambio cultural es un hecho" (35). El cambio cultural ha sido un hecho consciente de los pueblos alfabetizados desde la antigüedad. Por ejemplo, es bien sabido que las principales civilizaciones de la antigüedad tendían a idealizar una “edad de oro” ya desaparecida. Ésa es una concepción del cambio cultural. En lugar de hacer una afirmación tan deshistorizada, Bajtín podría haber comentado los diferentes "cronotopos" representados por diferentes gradaciones conceptuales del

${ }^{5}$ Para los fines de este argumento, en vez de traducir el neologismo de Bajtín "paзноречие [raznorechie] como "plurilingüismo", he preferido el término "heteroglosia," que es la versión en español de la muy conocida traducción al inglés de Caryl Emerson y Michael Holquist (1981), "heteroglossia", que me parece más descriptivo del concepto desarrollado por Bajtín de diferentes variedades lingüísticas en coexistencia dentro de un solo lenguaje; "plurilingüismo" se puede entender prima facie como el uso de múltiples idiomas dentro de un texto, lo cual no viene al caso. 
cambio histórico en diversas culturas literarias durante varios periodos, en la medida en que esos cronotopos pueden observarse en los discursos pertenecientes a esas culturas. Esto no quiere decir que Kaplan esté equivocada - en la modernidad, el cambio cultural sí es un hecho- sino que su afirmación parece estar despojada de cualquier diálogo con un contexto sociohistórico más amplio y extenso.

\section{Exilio y extrañamiento}

Sin embargo, este cambio cultural catalizado por las innovaciones formales centrífugas de Joyce no puede entenderse aislado de su historia de vida centrífuga, ni de su advocación del exilio utilizado como arma para el arte. Aunque Edward Said escribió extensamente sobre esta capacidad del exilio para ser un arma al servicio de la producción artística e intelectual, su posición permaneció algo ligada a la noción esencial y ahistórica del exilio estético que Kaplan ha deconstruido. Vale la pena, no obstante, examinar sus observaciones, por ejemplo, cuando escribe:

The exile [...] exists in a median state, neither completely at one with the new setting nor fully disencumbered of the old, beset with half-involvements and half-detachments, nostalgic and sentimental on one level, an adept mimic or a secret outcast on another. Being skilled at survival becomes the main imperative, with the danger of getting too comfortable and secure constituting a threat that is constantly to be guarded against. (Said, 1994: 49)

Kaplan presumiblemente señalaría el pathos del melancólico sentido de pérdida y nostalgia de Said y afirmaría que estos negativos se vuelven positivos para el propio Said, una puesta en escena de la pérdida calculada para conseguirle un grado de simpatía pública al ser un intelectual palestino en el exilio. Este aspecto positivo habría contribuido en última instancia a las trampas de su autoridad intelectual, particularmente en asuntos de exilio, movilidad, inmigración, diáspora, transnacionalismo, etcétera. Además, es análogo a las mejoras de reputación que los modernistas, particularmente los de la Generación Perdida, cosecharon de la 
difícil situación de su falta de hogar y es otro ejemplo de las economías de pérdida melancólica y ganancia estética en los topos de viaje que analiza Kaplan.

Dicho esto, sin embargo, la perspectiva más comprensiva de Said sobre el exilio, los viajes, la inmigración, etcétera, es al menos digna de respeto si la entendemos como la perspectiva de un exiliado y un inmigrante, y resulta que resuena fuertemente con el concepto de heteroglosia como una resistencia intelectual o estética a los factores centralizadores y monologizantes. Said (2001) escribe:

Even if one is not an actual immigrant or expatriate, it is still possible to think as one, to imagine and investigate in spite of barriers, and always to move away from the centralizing authorities towards the margins, where you see things that are usually lost on minds that have never traveled beyond the conventional and the comfortable. [...] The exilic intellectual does not respond to the logic of the conventional but to the audacity of daring, and to representing change, to moving on, not standing still. [...] Exiles cross borders, break barriers of thought and experience. (170)

Si bien las advertencias de Kaplan sobre la "melancolía nostálgica" que respalda tal perspectiva siguen vigentes, hay muchos ejemplos de los frutos de tales cruces de fronteras, especialmente del periodo modernista. Pero si el sujeto exílico es capaz de romper estas "barreras del pensamiento y la experiencia”, ¿cuáles son los mecanismos específicos por los que esto ocurre?

Para abordar esta pregunta, puede ser útil considerar bajo una nueva perspectiva el concepto formalista ruso de Viktor Shklovsky de "extrañamiento" (que también se traduce, aunque con menor precisión, como "desfamiliarización"). "Extrañamiento" es el término de Shklovsky para el proceso por el cual un escritor describe un hecho común como si fuera percibido por primera vez, como si no fuera ordinario sino "extraño". Él lo ve como una de las herramientas primarias del arte, argumentando que para hacer que una piedra se sienta "pétrea", a uno le debe de parecer extraña: "Al 'extrañar' los objetos y hacer compleja su forma, el dispositivo del arte hace que la percepción sea larga y “laboriosa” (Shklovsky, 
1990: 6). La noción de extrañamiento de Shklovsky toma como caso paradigmático lo que es familiar y no ajeno; primero ilustra la técnica con un ejemplo de Tolstoi, en el que el autor imagina que una forma familiar de castigo podría haber sido de otra manera:

In "Shame," Tolstoi enstranges the idea of flogging by describing people who, as punishment for violating the law, had been stripped, thrown down on the floor, and beaten with switches. [...] In a note on this passage, Tolstoi asks: "Just why this stupid, savage method of inflicting pain and no other: such as pricking the shoulder or some such other part of the body with needles, squeezing somebody's hands or feet in a vise, etc." [...] [this is] typical of the way Tolstoi reaches our conscience. (Shklovsky, 1990: 6)

Sin embargo, esto no significa que las cosas que ya son extranjeras no puedan también ser extrañadas. De hecho, el concepto de extrañamiento puede resultar útil para explicar un factor que ha llevado al éxito artístico del exiliado a lo largo de los siglos y en una miríada de entornos geográficos y sociales. Dado que el exiliado automáticamente mira con novedad todo lo que encuentra en su nuevo entorno, es decir, que todo le resulta más o menos extraño, el trabajo artístico de extrañamiento se vuelve, en consecuencia, más fácil de realizar. Por lo tanto, por un lado, la percepción natural del exiliado de la extrañeza sensorial y lingüística parece apta para conferir una ventaja creativa al hacer extrañas las cosas que son mundanas para los habitantes locales. Por otro lado, a medida que el exiliado se acostumbra a lo extranjero y lo desconocido, los recuerdos del hogar que alguna vez parecieron irrefutablemente ordinarios pueden apreciarse por sus peculiaridades, por su extrañeza recién recontextualizada.

Cabe mencionar aquí que Erich Auerbach (2013), en su Mimesis de 1946, sugiere que el realismo comenzó como exotismo, pero luego se lo llevó a casa, por así decirlo, para que lo familiar pudiera ser visto con nuevos ojos. Pero el modernismo lleva la extrañeza de lo familiar a un extremo sin precedentes, desarrollando lo que Francine Masiello (2004) llama "el tropo del secreto [que] funciona como 
un pivote o bisagra para equilibrar la relación entre los que saben y los que permanecen al margen" (55). Si bien este tropo ciertamente deriva en gran parte del elitismo intelectual del modernismo, la tendencia a codificar significados difíciles u ocultos bajo la superficie de un texto también puede originarse, al menos en algunos casos, en la experiencia expatriada del artista, quien de facto debe interpretar y decodificar significados ocultos o difíciles en los enigmas de una cultura extranjera. Así, la hipercomplejidad del modernismo puede, en algunos casos, representar una especie de respuesta reflexiva y defensiva a la experiencia perceptiva hipercompleja de enfrentarse a un entorno exílico y, por tanto, radicalmente desconocido. Entonces, si bien los críticos y los artistas pueden haber idealizado o esencializado el estado del exilio como propicio para la originalidad y la innovación artística, sugiero que también puede haber un mecanismo subyacente para el fenómeno. El comentario de Kaplan de que la puesta en escena de las miserias del exilio ha beneficiado la reputación de varios escritores (y críticos) modernistas es acertado; sin duda confiere una ventaja más en términos de simpatía e intriga del lector, una ventaja que en algunos casos puede tener dimensiones estéticas o afectivas. Su crítica, sin embargo, no logra negar o explicar la importancia del exilio como una potente fuente de inventiva estética o intelectual. Todavía hay fuerza en la extrañeza.

\section{Suciedad como arte}

Un ejemplo tanto del cambio cultural centrífugo como del tipo de extrañamiento estético facilitado por la experiencia del exilio sería la inclusión que hizo Joyce de material ostensiblemente obsceno en sus más grandes trabajos modernistas. En Ulysses en particular, estas inclusiones constituyen un estrato dialógico específico dentro de la novela, y también un estrato extranovelístico, tanto geográfico como histórico, que ejemplifica la heteroglosia en su forma más socialmente subversiva, más carnavalesca. Leo Bersani (1988) ha observado que el mismo título de la novela “alude a uno de los héroes más ingeniosos y astutos de la literatura mundial” y que el patronímico de Stephen Dedalus es el del "artífice astuto que, tanto construyó el 
laberinto de Minos como pudo escapar del mismo" (2). Por tanto, escribe Bersani (1988), "basta con echar un vistazo al título y leer la primera página de Ulysses para estar advertidos: el engaño y la astucia son las primeras connotaciones de la novela, por lo que se plantea desde el principio la posibilidad de que esas cualidades no sólo pertenezcan a ciertos personajes dentro del texto, sino que, más significativamente, éstos definan una estrategia autoral" (2). Joyce sabía que, si el contenido licencioso de su libro era fácilmente inteligible, nunca llegaría a los mercados cruciales de Inglaterra o Estados Unidos. Por lo tanto, tuvo que extrañar experiencias cotidianas, como la defecación, la masturbación y el sexo, a un grado tan radical que los censores no reaccionarían de la manera habitual; es decir, que encontrarían a Ulysses una experiencia de lectura tan difícil y arcana que no se sentirían ofendidos por ella de la manera a la que estaban acostumbrados. Y, de hecho, como concluyó el juez John M. Woolsey al levantar la prohibición de Estados Unidos en 1933,

"Ulysses no es un libro fácil de leer. Es brillante y aburrido, inteligible y oscuro en algunos momentos. En muchos lugares me parece repugnante, pero, aunque contiene, como he mencionado anteriormente, muchas palabras que se suelen considerar sucias, no he encontrado nada que considere suciedad por amor a la suciedad. [...] leer Ulysses en su totalidad, como un libro debe leerse en una prueba como ésta, no tiende a excitar impulsos sexuales o pensamientos lujuriosos" (Vanderham 1997: 116-117).

Entonces, la extrema desfamiliarización de Joyce de las palabras y acciones "sucias" constituyó una victoria para la heteroglosia en un sistema unitario estrictamente controlado de normas lingüísticas, pues esta extrañeza, esta "suciedad por amor al arte”, ayudó a habilitar el primer vehículo legal para tal material subido de tono y poder viajar de forma centrífuga a través de las redes de distribución del idioma inglés. Así, con la decisión de Woolsey, Ulysses asaltó la ciudadela de los mercados de habla inglesa, un polizón modernista "sucio" escondido en el caballo de Troya de su forma desfamiliarizada. Éste es uno de los momentos suprema- 
mente estéticos (y mitológicamente oportunos) en la historia de la heteroglosia literaria como fenómeno específicamente geográfico y temporal, y es uno de los factores más destacados que llevaron a Ulysses a convertirse en un parangón del modernismo internacional. En términos de Bajtín, es el Joyce centrífugo, tanto en el artista como en el arte, tanto en los estratos sucios como en los exaltados del discurso novelístico, el que más merece la atención de la teoría cultural actual.

\section{Forma centrífuga}

Otro excelente ejemplo tanto de innovación modernista como de extrañamiento inspirado por el exilio sería la corriente de conciencia joyceana o, preferiblemente, el monólogo interior, cuyo prototipo atribuyó Joyce a la novela simbolista de Édouard Dujardin de 1887, Les lauriers sont coupés. Este modo narrativo, manejado indistintamente con el discurso indirecto libre joyceano y la narración en tercera persona joyceana, lleva el dialogismo a alturas formales y temáticas inéditas, porque es un ejercicio de creación de una especie de sátira menipea inversa, un teatro inverso del absurdo en el que nosotros, el "público", somos puestos en diálogo tanto con un narrador evanescente como con los procesos de pensamiento, o soliloquios internos, de los "actores" de la obra. En la escena serenamente profana del retrete de "Calypso", esta táctica dialoga juguetonamente con el realismo grotesco de Leopold Bloom, las fuerzas de sus “estratos corporales inferiores”, con sus habilidades críticas y cognitivas como publicista y aspirante a literato. El siguiente pasaje describe a Bloom defecando mientras leía un cuento premiado en la revista Titbits escrito por Phillip Beaufoy, un escritor al que, según nos dicen, Bloom "envidiaba amablemente" (Joyce, 1990: 69). Comienza con una narración en tercera persona, luego oscila entre las tres principales modalidades de la narrativa joyceana y simultáneamente mantiene una oscilación del lenguaje en la que un estrato del discurso más unitario, unívoco y aceptable puede entenderse doblemente en un estrato tajantemente diferente (y más bajo): 
Quietly he read, restraining himself, the first column and, yielding but resisting, began the second. Midway, his last resistance yielding, he allowed his bowels to ease themselves quietly as he read, reading still patiently, that slight constipation of yesterday quite gone. Hope it's not too big bring on piles again. No, just right. So. Ah! Costive. One tabloid of cascara sagrada. Life might be so. It did not move or touch him but it was something quick and neat. Print anything now. Silly season. He read on, seated calm above his own rising smell. (Joyce, 1990: 69)

Es importante recordar que antes de Ulysses, los novelistas simplemente no incluían descripciones de experiencias tan íntimas y cotidianas como defecar leyendo una revista. Por lo tanto, esto debe tomarse como un ejemplo de realismo, y la honestidad en el arte que Gustave Courbet y Gustave Flaubert habían logrado unos ochenta años antes, llevada ahora a su extremo lógico. Pero, al mismo tiempo, lo que tenemos aquí es todo menos el estilo sencillo y directo que exigían las formas anteriores de realismo artístico y literario. Por ejemplo, el juego irónico con la "columna" yuxtapone dialógicamente las columnas emergentes de los excrementos verticales de Bloom con las columnas tipográficas del periódico con las que limpiará los restos de las primeras. Mientras asimilamos, "Quietly he read, restraining himself, the first column and, yielding but resisting, began the second", no se puede saber de cuál de los dos tipos de columna se está hablando: mierda o premio, cuando se refiere a ésta como "comenzó la segunda”. Una lectura centrípeta nos guiaría a asumir que esta columna es la literaria, pero bien podría ser de la variedad fecal, especialmente dado el contexto (y el autor). Mientras Bloom continúa leyendo y defecando, el estilo de narración cambia de tercera persona a estilo indirecto libre: "Midway, his last resistance yielding, he allowed his bowels to ease themselves quietly as he read, reading still patiently, that slight constipation of yesterday quite gone”. La última frase está escrita en estilo indirecto libre, porque la frase "that slight constipation of yesterday quite gone" proviene del punto de vista de Bloom, de la memoria de Bloom. Por el contrario, si la narración de ese recuerdo se hubiera transmitido en tercera persona, habría utilizado necesaria- 
mente una construcción sin perspectiva como " $a$ slight constipation," en lugar de comenzar con el determinante demostrativo: "that slight constipation".

Este cambio de ritmo narrativo establece magistralmente la entrada completa del párrafo en el monólogo interior: "Hope it's not too big bring on piles again. No, just right. So. Ah! Costive. One tabloid of cascara sagrada. Life might be so”. Aquí nuevamente hay una mezcla hábilmente ambigua de posibles estratos de significación: Bloom se está refiriendo evidentemente al tamaño de sus excrementos cuando le preocupa que éstos le producirán hemorroides, pero cuando llega a "Ah! Costive. One tabloid of cascara sagrada. Life might be so", se puede entender que su discurso interno combina lecturas de los estratos superior e inferior de este pasaje una vez más, aunque desde un "orden” observacional bastante diferente, para usar la terminología de Niklas Luhmann (2000). El término "costive" (estreñido) se deriva del latín “constipare” y puede referirse al estreñimiento, pero aquí también podría referirse a obras literarias que son "lentas", "difíciles" o "poco comunicativas”. Al principio, podríamos pensar que Joyce está sugiriendo sarcásticamente que Bloom encuentra la historia de Beaufoy lenta o inaccesible, pero esta lectura se cancela inmediatamente por el cambio abrupto que regresa a la narración en tercera persona: "It did not move or touch him but it was something quick and neat”. Una vez más, esto puede entenderse como una descripción de la historia que está siendo leída o, en la misma medida, como el paso de los excrementos de Bloom, ahora sin estreñimiento gracias a que tomó un laxante de "cáscara sagrada”. Más importante, sin embargo, su movimiento oloroso nos remite a la palabra “Costive", un término que probablemente no describe el estilo de Beaufoy, pero que puede estar, y ha estado, asociado con los múltiples estilos del propio Ulysses, como se captura en este mismo pasaje: "lento", "difícil”, "poco comunicativo". Esta posible autorreferencia metatextual está respaldada por la ponderación filosófica aparentemente discordante de Bloom, "Life might be so", ya que su propia "vida" como personaje literario, y la vida de todo Dublín en ese día, está en proceso de ser capturado y hecho vivo en esta novela "costosa”.

La posibilidad de un guiño joyceano al estilo inspirado de Ulysses en relación con lo que está leyendo Bloom también es consistente con el hecho de que la 
única frase incluida en Ulysses de la historia de Beaufoy, aunque no es "costosa", es identificada por Gifford (1988) como "la broma privada de Joyce a expensas de su propia adolescencia. Aparentemente, Joyce escribió una historia, destinada a hablar de Titbits (Golosinas) y dinero, que incluía la frase que Bloom lee" (81), que es "Matcham often thinks of the masterstroke by which he won the laughing witch who now" (Joyce, 1990: 69). En otras palabras, es muy posible que Joyce esté comparando su estilo adolescente (ni "emocionante" ni "conmovedor", sino "rápido y pulcro") con su estilo maduro en Ulysses, uno que sin duda es "estreñido", pero de todos modos posible de acceder a través de una especie de "cáscara sagrada", o corteza sagrada, que el lector de Ulysses deberá consumir para conmoverse y emocionarse con esta novela y su autor. Y esto configura hábilmente las siguientes frases que oscilan entre el monólogo interior y la narración en tercera persona: "Print anything now. Silly season. He read on, seated calmly above his own rising smell”. El realismo rabelaisiano de Joyce subvierte el lenguaje unitario de la ficción vulgar fusionándolo y confundiéndolo con el lenguaje interno del sujeto que lee dialógicamente. Esta muestra de monólogo interior joyceano constituye, por tanto, una dramatización exquisitamente cochina de la tensión bajtiniana entre monoglosia (ficción inculta) y heteroglosia (el lenguaje interno primordial de los estratos inferiores); es decir, entre las fuerzas centrípeta y centrífuga en la vida del lenguaje novelístico.

\section{Forma exílica, afecto estético}

Por supuesto, es necesario observar que en Ulysses no es todo suciedad. En otras ocasiones, también absorbe los estratos más altos del discurso abstracto extra-novelístico. Por ejemplo, Ulysses ha llegado a simbolizar el tropo del exilio modernista, en gran parte como resultado de la relación dialógica entre un Leopold Bloom errante, un Judío errante, un Odiseo errante y un Joyce errante. Pero, como señala Franco Moretti (1996), la importancia del autor en todo esto da paso a la importancia del "pluralismo estilístico" de la novela, que Moretti ve como un "signo de la importancia de Mijaíl Bajtín” para la teoría cultural (182-83). Por ejemplo, Ulysses ejemplifica 
la tendencia de la heteroglosia hacia la parodia, muy comentada por Bajtín, ya que es burlesca y subvierte todo tipo de géneros discursivos, que van desde la crítica literaria hasta la liviandad de las revistas femeninas y la propia tradición épica. Moretti (1996) simpatiza con la idea de que Ulysses tiene una importancia fundamentalmente formal en la historia de la novela, ilustrándola figurativamente:

Exagerando un poco, podríamos decir que los protagonistas de Ulysses no son Stephen Dedalus y la pareja Bloom, sino dos técnicas -la corriente de la conciencia y la polifonía- que se entrelazan y chocan a lo largo de la novela, en una especie de drama técnico en el que el estilo inicial de la novela (la corriente de la conciencia) es poco a poco flanqueado, desafiado y finalmente derrotado por el recién llegado, que acaba imponiéndose como amo del universo joyceano. (183)

Sin embargo, apreciar sólo los aspectos formales, estéticos y técnicos de Ulysses sería perder gran parte de lo que hace que el trabajo de Joyce sea valioso para la teoría cultural. Por ejemplo, para muchos lectores se evoca una dimensión afectiva al reconocer que Joyce fue un escritor irlandés cuya determinación de permanecer expatriado toda su vida sigue contribuyendo fuertemente a la desestabilización de la noción de literaturas "nacionales". De hecho, este aspecto del trabajo de Joyce ha sido el foco de los intentos por aplicar la teoría bajtiniana al estudio de Ulysses a lo largo de líneas poscoloniales, minimizando los elementos estéticos "alegres" de la novela. Como escribe Eric D. Smith (2002), por ejemplo, "la presentación de Joyce del cuerpo poscolonial fragmentado (la novela como Irlanda) no es una participación gozosa en la cosificación moderna, sino más bien una acusación seria de ella, una protesta dialógica de la voz del Otro reprimido del modernismo" (132). Sin embargo, aunque el Joyce serio, portentoso y poscolonial es ciertamente un Joyce a ser tomado en cuenta, ${ }^{6}$ tiene poco sentido defenestrar, en el camino,

${ }^{6}$ Para un estudio en profundidad sobre el poscolonialismo en Joyce desde un punto de vista bajtiniano, véase Chou (2002). 
al brillante "dramaturgo técnico" que Moretti describe: el jocoso y alegremente insolente Joyce.

De hecho, a veces los dos van de la mano. En "Scylla and Charybdis", Stephen hace una especie de argumento proto-neo historicista sobre la intersección de la propiedad, la historia y la estética en las obras de Shakespeare, un argumento que "viaja" por todo el mundo:

- The sense of beauty leads us astray, said beautifulinsadness Best to ugling Eglinton.

Steadfast John replied severe:

-The doctor can tell us what those words mean. You cannot eat your cake and have it.

-Sayest thou so? Will they wrest from us, from me, the palm of beauty?

-And the sense of property, Stephen said. He drew Shylock out of his own long pocket. The son of a maltjobber and moneylender he was himself a cornjobber and moneylender with ten tods of corn hoarded in the famine riots. His borrowers are no doubt those divers of worship mentioned by Chettle Falstaff who reported his uprightness of dealing. He sued a fellowplayer for the price of a few bags of malt and exacted his pound of flesh in interest for every money lent. [...] All events brought grist to his mill. [...] The lost armada is his jeer in Love's Labour Lost. His pageants, the histories, sail fullbellied on a tide of Mafeking enthusiasm. [...] The Sea Venture comes home from Bermudas and the play Renan admired is written with Patsy Caliban, our American cousin. The sugared sonnets follow Sidney's. As for fay Elizabeth, otherwise carroty Bess, the gross virgin who inspired The Merry Wives of Windsor, let some meinherr from Almany grope his life long for deep-hid meanings in the depth of the buckbasket. (Joyce, 1990: 204-05)

Inmediatamente es evidente la observación de Stephen, basada, según Gifford (1988: 234-235), en relatos historiográficos que Joyce había leído, de que Shakespeare parasita todo y a todos, desde su "compañero de juego" de quien "extrajo su libra 
de carne", hasta eventos históricos "creando" sentimientos nacionalistas por sus "rebosantes" obras históricas, o al naufragio narrativo del Sea Venture para The Tempest, o bien las innovaciones poéticas de Sidney para los sonetos. Sin embargo, Stephen también teje en esta letanía de los robos de Shakespeare algunos "significados profundamente escondidos" de su propia cosecha, significados que sólo pueden ser desentrañados siguiendo sus sutiles hilos de referencia. Consideremos, por ejemplo, la oración "The Sea Venture comes home from Bermudas and the play Renan admired is written with Patsy Caliban, our American cousin”. The Tempest, de Shakespeare, para la que Ernst Renan escribió una secuela, se basó parcialmente en la narrativa del naufragio de la tripulación del Sea Venture, un barco que se hundió en las Bermudas durante un viaje a América en 1609. "Patsy", según explica Gifford (1988), se refiere a las "escenas caricaturescas de los inmigrantes irlandeses del siglo XIX" en los Estados Unidos. Finalmente, Our American Cousin es la comedia de Tom Taylor de 1858 que Abraham Lincoln estaba viendo la noche en que fue asesinado. Entonces, en una sola oración, Stephen imita la intersección de propiedad, historia y estética que defiende en la obra de Shakespeare al comparar la situación de los campesinos inmigrantes irlandeses en América ("Patsies") con los nativos colonizados de Bermudas ("Calibans"), y a los esclavos afroamericanos por quienes Lincoln perdió la vida ("nuestros primos estadounidenses"). Así, la observación histórica de que cada uno de estos pueblos oprimidos ha compartido el estatus de "propiedad", de una forma u otra, se condensa en la figura polifónica del caníbal indígena irlandés y negro de Shakespeare: "Patsy Caliban, nuestro primo americano". ${ }^{7}$

Cabe destacar que este comentario lo hace Stephen en respuesta a una pregunta "wildeana” sobre los propósitos contradictorios de la moralidad y la belleza en el arte: "Will they wrest from us, from me, the palm of beauty?" o como lo expresó Gifford (1988), “quitárselo al artista y dárselo al moralista?” (234). La respuesta es otra oscilación: ambos y ninguno. En la mezcla intrincada de signifi-

${ }^{7}$ Para un análisis bajtiniano más extenso de la relación dialógica entre Joyce y Shakespeare, véase Booker (1995). 
cados de Stephen, vemos al Joyce poscolonial comentando con brillante oblicuidad técnica el trágico precio que pagó Lincoln por emancipar a los esclavos, por abolir el "sentido de propiedad" que los gobernaba de la misma manera en que Próspero gobernó a Calibán, y como el monarca inglés gobierna a los irlandeses. Stephen así forja el título del objeto de arte que chocó con la historia, Our American Cousin, en una especie de metempsicosis intertextual, a través de la vinculación literaria, de pueblos "primos" oprimidos en sus distintas formas de "propiedad". Creo que este puede ser uno de los "significados profundamente escondidos en el fondo de la canasta" (Joyce, 1990: 205) de este párrafo, y una de las formas en que los estratos políticos y estéticos del discurso de Joyce se manifiestan dialógicamente, sin contraerse en un cierre ideológico tendencioso.

Este artículo dio inicio preguntándose si un novelista que Bajtín notablemente omitió podría llegar a producir la máxima expresión de los propios ideales de representación novelística de Bajtín. Porque Ulysses es una novela que, como ninguna otra, es dialógica, carnavalesca, multicronotópica, corpocéntrica y lingo-culturalmente interestratificada. Para reforzar este argumento, el principal concepto bajtiniano de heteroglosia se expandió más allá de su original ámbito intranovelístico original, para describir tanto las energías como las tensas complejidades del exilio como motor del arte modernista. Una propuesta de un eje teórico que vincula el exilio, la heteroglosia y la noción de extrañamiento de Shklovsky llevó a una discusión sobre la obscenidad como un objeto centrífugo y estético para Joyce: la suciedad por amor al arte. Y esto, a su vez, permitió un análisis de las capas de representación interestratificadas en Ulysses como una forma de heteroglosia cuya fuerza depende tanto del elemento exílico como del elemento afectivo. Si efectivamente Kaplan es astuta al señalar que el exilio modernista recicla una economía de ganancia estética a cambio de una pérdida melancólica, podemos decir con Said que algunos participantes de esa economía, Joyce en particular, logran una centrifugalidad radical que desafía y trasciende las fronteras implicadas por esa crítica, rompiendo las "barreras del pensamiento y la experiencia" (Said, 2001: 170) que sólo permite el arte del exilio. 


\section{REFERENCIAS BIBLIOGRÁFICAS}

AUERBACH, Erich. (2013). Mimesis: The Representation of Reality in Western Literature. Princeton University Press.

AZAREDO, Genilda. (2020). "Eveline e Elvira: subjetividades femininas em diálogo intertextual”. Revista Artemis, 29(1), 316-325.

BajTín, Mijaíl. (1984). Problems of Dostoevsky's Poetics. University of Minnesota Press.

BAjTín, Mijaíl. (1989). Teoría y estética de la novella (Helena S. Kriúkova y Enrique Cazcarra, Trads.). Altea; Taurus; Alfaguara.

BAJTíN, Mijaíl. (1996-2012). Sobranie sochinenii v semi tomakh [Obras en 7 volúmenes] (S. G. Bocharov, N. I. Nikolaev y L. S. Melikhova, Eds.). Russkie slovari Iazyki slavianskikh kul'tur Publ.

BALKAYA, Mehmet Akif. (2017). "Voices in James Joyce's 'The Dead': A Bakhtinian Reading". Fournal of International Social Research, 10(52), 69-74.

BERSANI, Leo. (1988). "Against Ulysses". Raritan: A Quarterly Review, 8(2), 1-32.

BOOKER, M. Keith. (1995). Foyce, Bakhtin, and the Literary Tradition: Toward a Comparative Cultural Poetics. University of Michigan Press.

BRADBURY, Malcolm. (1991). "The Cities of Modernism”. In Malcolm Bradbury and James McFarlane (Eds.), Modernism: 1890-1930. Penguin Books.

CANEDA CABrerA, M. Teresa. (1996). "Ulysses and Heteroglossia: A Bakhtinian Reading of the 'Nausicaa' Episode”. Revista Alicantina de Estudios Ingleses 9(1), 33-40.

CHOU, Hsing-Chun. (2002). Foyce, Bakhtin, and Postcolonial Trialogue: History, Subjectivity, and the Nation in Ulysses. (Tesis de doctorado, University of Glasgow). Recuperado de http://theses.gla.ac.uk/1800/

CLARK, Katerina; HOLQUiST, Michael. (1984). Mikhail Bakhtin. Harvard University Press.

GEORGE, Jibu Mathew. (2017). "James Joyce and the 'Strolling Mort': Significations of Death in Ulysses". Mortality, 22(1), 60-74. https://doi.org/10.1080/13576275.20 $\underline{16.1204276}$ 
GIFFORD, Don (with SEIDMAN, RobertJ). (1988). Ulysses Annotated: Notes for Fames Foyce's Ulysses. University of California Press.

JOYCE, James. (1990). Ulysses. Vintage International.

KAPLAN, Caren. (1996). Questions of Travel: Postmodern Discourses of Displacement. Duke University Press.

KERSHNER, R. B. (1989). Foyce, Bakhtin, and Popular Literature : Chronicles of Disorder. The University of North Carolina Press.

KIM, Kyoungsook. (2017). "The Carnival of the Repressed Others: Re-Reading Joyce's 'Circe' through Bakhtin”. Fames foyce fournal, 23(2), 25-31. http://www. joycesociety.or.kr/data/thesis/11977075292425.pdf

LUHMAnN, Niklas. (2000). Art as a Social System. Stanford University Press.

MASIELlO, Francine. (2004). "Joyce in Buenos Aires (Talking Sexuality through Translation)". Diacritics, 34(3/4), 55-72.

MORETTI, Franco. (1996). The Modern Epic: The World System from Goethe to García Márquez. Verso.

PRATT, Mary Louise. (1992). Imperial Eyes: Travel Writing and Transculturation. Routledge.

SAID, Edward. (1994). Representations of the Intellectual: The 1993 Reith Lectures. Pantheon.

SAID, Edward. (2001). Reflections on Exile. Granta Publications.

SHKLOVSKY, Viktor. (1990). Theory of Prose. Dalkey Archive Press.

SMiTH, Eric D. (2002). "I Have Been a Perfect Pig”: A Semiosis of Swine in 'Circe”". Foyce Studies Annual, (13), 129-146. https://doi.org/10.1353/joy.2002.0010

TEKDEMIR, Hande. (2017). “Textual Conversations and Dialogic Imagination in James Joyce's Ulysses”. Celal Bayar University fournal of Social Sciences, 15(1), 131-146. https://doi.org/10.18026/cbayarsos.297878

VAnderham, Paul. (1997). Fames Foyce and Censorship: The Trials of Ulysses. New York University Press. 